\title{
Is there a role for pharmacogenetics in the treatment of panic disorder?
}

\author{
"To date, little research is available on the pharmacogenetics of panic \\ disorder, with inconsistent results."
}

Keywords: genetic $\bullet$ panic disorder $\bullet$ polymorphism $\bullet$ serotonin

\section{Pharmacogenetics \& personalized psychiatry}

The ultimate goal of a personalized approach to pharmacotherapy is discovering reliable predictors of psychotropic drug response/tolerability to tailor pharmacotherapy according to each patient's unique features. The interest in this area emerged for several reasons.

First, the evidence-based pharmacological guidelines for psychiatric disorders, including panic disorder (PD), are mainly based on the results of randomized clinical trials (RCTs). They are carried out in highly selected populations of patients and refer to the 'average' ideal patient who does not represent real-life patients with their unique characteristics. Consequently, in clinical practice we still proceed by a 'trial-error' approach without any evidence-based predictor or test to guide the choice.

Second, while antidepressant drugs are recommended for depressive and anxiety disorders, their clinical outcomes are unsatisfying. The interindividual variability in antidepressant efficacy and induced side effects is high, with many patients not obtaining beneficial responses and/or discontinuing treatments due to adverse side effects.

PD is not an exception. It is a common (lifetime prevalence 3-4\%), disabling anxiety disorder including unexpected panic attacks (PAs), anticipatory anxiety and phobic behaviors. Recommended drugs do not achieve full remission in $20-40 \%$ of patients and additional cognitive behavioral therapy does not fill this gap. The rate of relapses within 6 months after drug discontinuation is $25-50 \%$ and the picture is not better for the rate of patients with residual panic-phobic symptoms (up to $50 \%$ ) [1] or who discontinue treatments for side effects (20-30\%) [2].

Among multiple factors affecting pharmacotherapy efficacy/tolerability, genetic variability plays a relevant role. Familial and twin studies supported a significant genetic contribution to antidepressants' efficacy and metabolism/distribution [3]. Recently, 42\% of individual divergences in antidepressant response was attributed to genetic variations [4]. Pharmacogenetics, in other words, the study of how inherited genetic variations affect an individual's response to medications or tolerability, may contribute to the development of personalized treatments, as already well established in oncology [5]. So far, pharmacogenetics were mainly studied in depression and, despite some inconsistencies, polymorphisms of serotonergic and nonserotonergic (brain-derived neurotrophic factor; P-glycoprotein) genes seemed to be involved in selective serotonin reuptake inhibitors (SSRIs) efficacy/tolerability [4]. Several kits for pharmacogenetic tests are commercially available, but official guidelines for their use are still lacking. Recently, US FDA approved Roche AmpliCyp CYP450 test as a tool to assesses functional genetic polymorphisms of the cytochrome $\mathrm{P} 450$ CYP2D6/CYP2C19 genes, involved in antidepressants metabolism, since dose adjustments according to metabolizer phenotype may contribute to reduce the side effects of antidepressants [5]. Though clinical applications are still limited, preliminary studies showed advantageous cost-benefit ratio of genetic-guided antidepressant treatments [4].

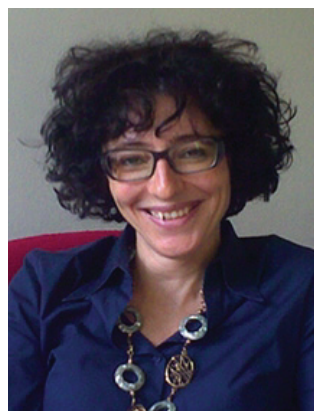

Daniela Caldirola

Author for correspondence: Department of Clinical Neurosciences, Villa San Benedetto Menni, Hermanas Hospitalarias, Via Roma 16, 22032, FoRiPsi, Albese con Cassano, Como, Italy Tel.: +39031 4291511

Fax: +39031427246 caldiroladaniela@gmail.com

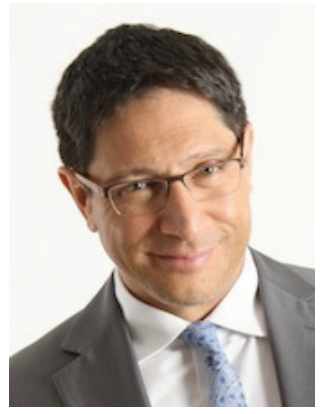

Giampaolo Perna

Department of Clinical Neurosciences, Villa San Benedetto Menni, Hermanas Hospitalarias, Via Roma 16, 22032, FoRiPsi, Albese con Cassano, Como, Italy and

Department of Psychiatry \& Neuropsychology, Faculty of Health, Medicine \& Life Sciences, University of Maastricht, Maastricht, The Netherlands and

Department of Psychiatry \& Behavioral Sciences, Leonard Miller School of Medicine, University of Miami, 1600 NW 10th Ave \#1140, Miami, FL 33136, USA

Future $\%$ fsg 


\section{Where are we now in PD?}

Compared to depression, anxiety disorders received less attention. To date, little research is available on the pharmacogenetics of $\mathrm{PD}$, with inconsistent results. Pharmacogenetic approach investigates genetic variants selected on the basis of 'a priori' hypotheses derived from preclinical/clinical findings [3]. A link between serotonin (5-HT) system and PD has been repeatedly supported: SSRIs are the first-line pharmacological intervention [1]; experimental manipulations of 5-HT system influenced the laboratory-induced PAs in subjects with PD [1]; susceptibility for PD was associated with polymorphisms of serotonin transporter (SLC6A4) [6] and 5-HT2A/5-HT1A receptors [7] genes; panic symptoms severity was associated with 5 -HT2A receptor and serotonin transporter promoter (5-HTTLPR) polymorphisms [7,8]. Thus, pharmacogenetic studies in PD focused on serotonergic candidate genes. We investigated the functional polymorphisms of the 5-HTTLPR in Caucasian patients with PD. Females homozygotes for the long variant $(I / l)$ and heterozygotes $(l / s)$ had greater decrease of PAs after 12-weeks paroxetine treatment than female homozygotes for the short variant $(s / s)$, whereas the groups did not differ in the improvement of anticipatory anxiety/phobic avoidance [9]. Conversely, in Japanese patients, the homozygotes $(s / s)$ reported greater reduction of panic-phobic symptoms than heterozygotes $(l / s)$, without gender differences, after 2 -week paroxetine treatment [10]. In a different Japanese sample, heterozygotes $(\mathrm{l} / \mathrm{s})$ showed a higher tendency to discontinue therapy for adverse side effects than homozygotes $(s / s)$, within the first 2 weeks of paroxetine treatment [2]. In two other Asian samples, no effects of 5-HTTLPR polymorphisms were found on panic-phobic symptoms or clinical global improvement after 4 weeks or 10-week paroxetine treatment, respectively [11,12]. In these studies, no effects of tryptophan hydroxylase (a rate-limiting enzyme in 5-HT biosynthesis) polymorphisms were also found [12], while patients with the 1019C/C genotype in the regulatory region of the 5-HT1A receptor gene showed a better clinical outcome than those with the C/G or G/G genotype [11]. Similarly, in a Caucasian sample, the G/G genotype was associated with a minimal decrease of PAs after 6-weeks paroxetine/sertraline treatment, while no relationship with 5-HTTLPR polymorphisms was found [13]. Finally, preliminary results of our group did not show association between 12-weeks paroxetine treatment response and functional polymorphisms of COMT (an enzyme involved in 5-HT/other monoamines catabolism) [14], while Korean patients with $L / L C O M T$ genotype had poorer clinical global improvement after 12-week paroxetine treatment [15]. These findings are insufficient to draw conclusions. The studies are few, with short-term follow- up, and the sample sizes are small with poor statistical power. Ethnicity of the samples may partly explain discrepancies in results, as shown in previous studies where the same allele of 5-HTTLPR was predictor of different SSRIs outcome/tolerability in Caucasian or Asian depressed patients [4]. Dose and duration of pharmacotherapy were heterogeneous as well as the inclusion criteria, evaluation time points and measures of outcome, thus making the findings hardly comparable. Finally, some studies used inappropriate outcome measures for PD, such as clinical global improvement, or total panicphobic symptoms score that does not disentangle drug effects on different clinical features of PD.

$$
\begin{aligned}
& \text { "...pharmacogenetics of panic disorder is } \\
& \text { at a very early stage but methodological } \\
& \text { improvements and promising innovative } \\
& \text { strategies may produce reliable advances in } \\
& \text { this field." }
\end{aligned}
$$

\section{Future perspective}

Much more efforts are clearly required in pharmacogenetics of PD and many challenging issues need to be considered. The candidate gene approach has the advantage to be based on putative neurobiological relevance of selected genes, but has some critical points:

- The neurobiology of PD is not fully understood and probably involves an interplay between genetic (half of the variance) and environmental factors [16];

- The mechanisms of actions of antipanic drugs are not fully clarified;

- The antipanic effects are probably influenced by multiple genetic variants with small effect size and potential additive/interactive effects that are not captured by studies with single or few candidate genes in small samples, as those conducted so far;

- Genetic variations are unlikely to completely explain divergences in individual responses to antidepressants. Environmental factors and gene-environment interactions may influence the pharmacological phenotypes [17];

- PD is a heterogeneous condition that includes different clinical phenomena probably resulting from distinct neurobiological mechanisms. Standard diagnostic criteria for PD do not reflect this complexity and the lack of biomarkers/endophenotypes in pharmacogenetic studies may significantly affect the results [1];

- The lack of standardized methodological criteria and the use of unsuitable measures of outcome may influence the reliability of the findings and mask potential promising results [18]. 
Future strategies to fill the gaps include:

- Multicenter collaborations for large prospective studies with standardized designs, higher statistical power and careful consideration of stratification factors (e.g., age, gender, ethnicity, previous pharmacological treatment);

- Expanding the investigated genes and studying the gene $x$ gene interactions. Connections between multiple polymorphisms of the serotonin system genes as well as between serotonergic genes and novel candidate genes may be relevant to the SSRIs antipanic effects. In animal models, pharmacological modulation of orexin and glutamatergic receptors may influence the occurrence of PAs and preliminary associations between human PD and genes of orexine/glutamatergic systems were found [1]. Since connections between serotonin and orexine/glutamatergic systems exist, the joined genetic investigation of these systems may produce more informative results on the individual response to SSRIs. Finally, the use of inbred strains with different genetic background in animals models of panic may suggest allelic variations of interest that could be tested in human pharmacogenetic studies;

- Implementation of a biomarker/endophenotype approach to decrease the variability within the nosographic diagnosis of PD. Hypersensitivity to hypercapnia is thought to be an endophenotype of panic, is strongly influenced by genetic factors and is associated with respiratory symptoms, higher frequency of PAs and familiarity for PD, possibly typifying a respiratory-panic subtype. Several abnormalities in respiratory/autonomic/balance system functions were found in patients with PD, that may result in different clinical symptoms and outcomes [1]. Incorporating the respiratory endophenotype or patterns of neurobiological functions in pharmacogenetic studies may help to select truly homogeneous patients, to identify more appropriate outcome measures and unravel specific pharmacogenetic associations;

- Connecting genome-wide association studies (GWAS) with pharmacological investigations (pharmacogenomics). GWAS are 'hypothesis free' and simultaneously genotype many hundred thousands of polymorphisms across the genome, allowing to surmount the need of an a priori hypothesis of the candidate genes approach. To date, no published pharmacogenomics studies in PD are available. Since this approach also has limitations $[3,17]$, the opportunity of combining GWAS with complementary strategies may be considered in designing future studies in PD. As GWAS identify genetic regions of interest, resequencing these regions taking into account the results from candidate genes studies may help to validate more relevant variants. The application of pathway analyses (i.e., the analyses of a set of variants within genes implicated in the same biological process) may increase the possibility to investigate the interactions between multiple genetic variants and to identify polymorphisms with single small effects that do not reach genomewide significance threshold. Finally, incorporating endophenotypes/neurobiological functions in pharmacogenomic studies may produce more reliable results;

- Considering stressful life events/early trauma. Interactions between serotonergic genes and adverse experiences in modulating antidepressant outcome of depressed patients were found, partly related to epigenetic mechanisms [19]. Since childhood maltreatment was significantly reported in patients with PD [20], gene-environment interactions may play a relevant role in pharmacogenetics of $\mathrm{PD}$.

In conclusion, pharmacogenetics of PD is at a very early stage but methodological improvements and promising innovative strategies may produce reliable advances in this field.

\section{Financial \& competing interests disclosure}

The authors have no relevant affiliations or financial involvement with any organization or entity with a financial interest in or financial conflict with the subject matter or materials discussed in the manuscript. This includes employment, consultancies, honoraria, stock ownership or options, expert testimony, grants or patents received or pending, or royalties.

No writing assistance was utilized in the production of this manuscript.

\section{References}

1 Perna G, Schruers K, Alciati A, Caldirola D. Novel investigational therapeutics for panic disorder. Expert Opin. Investig. Drugs 24(4), 491-505 (2015).

2 Aoki A, Ishiguro S, Watanabe T et al. Factors affecting discontinuation of initial treatment with paroxetine in panic

disorder and major depressive disorder. Neuropsychiatr. Dis. Treat. 10, 1793-1798 (2014).

3 Fabbri C, Di Girolamo G, Serretti A. Pharmacogenetics of antidepressant drugs: an update after almost 20 years of research. Am. J. Med. Genet. B. Neuropsychiatr. Genet. 162B(6), 487-520 (2013). 
4 Fabbri C, Minarini A, Niitsu T, Serretti A. Understanding the pharmacogenetics of selective serotonin reuptake inhibitors. Expert Opin. Drug Metab. Toxicol. 10(8), 1093-1118 (2014).

5 Hamilton SP. The promise of psychiatric pharmacogenomics. Biol. Psychiatry 77(1), 29-35 (2015).

6 Strug LJ, Suresh R, Fyer AJ et al. Panic disorder is associated with the serotonin transporter gene (slc6a4) but not the promoter region (5-httlpr). Mol. Psychiatry 15(2), 166-176 (2010).

7 Unschuld PG, Ising M, Erhardt A et al. Polymorphisms in the serotonin receptor gene $h t r 2 a$ are associated with quantitative traits in panic disorder. Am. J. Med. Genet. B. Neuropsychiatr. Genet. 144B(4), 424-429 (2007).

8 Lonsdorf TB, Ruck C, Bergstrom J et al. The symptomatic profile of panic disorder is shaped by the 5-httlpr polymorphism. Prog. Neuropsychopharmacol. Biol. Psychiatry 33(8), 1479-1483 (2009).

9 Perna G, Favaron E, Di Bella D, Bussi R, Bellodi L. Antipanic efficacy of paroxetine and polymorphism within the promoter of the serotonin transporter gene. Neuropsychopharmacology 30 (12), 2230-2235 (2005).

10 Saeki Y, Watanabe T, Ueda M et al. Genetic and pharmacokinetic factors affecting the initial pharmacotherapeutic effect of paroxetine in japanese patients with panic disorder. Eur. J. Clin. Pharmacol. 65(7), 685-691 (2009).

11 Ishiguro S, Watanabe T, Ueda M et al. Determinants of pharmacodynamic trajectory of the therapeutic response to paroxetine in Japanese patients with panic disorder. Eur. J. Clin. Pharmacol. 67(12), 1213-1221 (2011).

12 Kim W, Choi YH, Yoon KS, Cho DY, Pae CU, Woo JM. Tryptophan hydroxylase and serotonin transporter gene polymorphism does not affect the diagnosis, clinical features and treatment outcome of panic disorder in the korean population. Prog. Neuropsychopharmacol. Biol. Psychiatry 30(8), 1413-1418 (2006).

13 Yevtushenko OO, Oros MM, Reynolds GP. Early response to selective serotonin reuptake inhibitors in panic disorder is associated with a functional 5-ht1a receptor gene polymorphism. J. Affect. Disord. 123(1-3), 308-311 (2010).

14 Favaron E, Biffi S, Grassi M, Bellodi L, Perna G. Response to paroxetine and catecol-o-metil transferasi (comt) polymorphisms in panic disorder. Presented at: 11th National Congress of the Italian Society of Pyschopathology. Rome, Italy, March 2006.

15 Woo JM, Yoon KS, Choi YH, Oh KS, Lee YS, Yu BH. The association between panic disorder and the $1 / 1$ genotype of catechol-o-methyltransferase. J. Psychiatr. Res. 38(4), 365-370 (2004)

16 Schumacher J, Kristensen AS, Wendland JR, Nothen MM, Mors O, McMahon FJ. The genetics of panic disorder. J. Med. Genet. 48(6), 361-368 (2011).

17 Fabbri C, Porcelli S, Serretti A. From pharmacogenetics to pharmacogenomics: the way toward the personalization of antidepressant treatment. Can. J. Psychiatry 59(2), 62-75 (2014).

18 Narasimhan S, Lohoff FW. Pharmacogenetics of antidepressant drugs: current clinical practice and future directions. Pharmacogenomics 13(4), 441-464 (2012).

19 Reynolds GP, Mcgowan OO, Dalton CF. Pharmacogenomics in psychiatry: the relevance of receptor and transporter polymorphisms. Br. J. Clin. Pharmacol. 77(4), 654-672 (2014).

20 Perna G, Alciati A, Prestia D, Torti T, Nemeroff CB. Is there a link between child abuse and neglect ans anxiety disorders? Minerva Psichiatrica 54(2), 137-148 (2013). 